\title{
Inhibitory Control in High-Functioning Autism: Decreased Activation and Underconnectivity in Inhibition Networks
}

\author{
Rajesh K. Kana, Timothy A. Keller, Nancy J. Minshew, and Marcel Adam Just
}

Background: Inhibiting prepotent responses is critical to optimal cognitive and behavioral function across many domains. Several behavioral studies have investigated response inhibition in autism, and the findings varied according to the components involved in inhibition. There has been only one published functional magnetic resonance imaging (fMRI) study so far on inhibition in autism, which found greater activation in participants with autism than control participants.

\begin{abstract}
Methods: This study investigated the neural basis of response inhibition in 12 high-functioning adults with autism and 12 age- and intelligence quotient (IQ)-matched control participants during a simple response inhibition task and an inhibition task involving working memory.

Results: In both inhibition tasks, the participants with autism showed less brain activation than control participants in areas often found to be active in response inhibition tasks, namely the anterior cingulate cortex. In the more demanding inhibition condition, involving working memory, the participants with autism showed more activation than control participants in the premotor areas. In addition to the activation differences, the participants with autism showed lower levels of synchronization between the inhibition network (anterior cingulate gyrus, middle cingulate gyrus, and insula) and the right middle and inferior frontal and right inferior parietal regions.
\end{abstract}

Conclusions: The results indicate that the inhibition circuitry in the autism group is activated atypically and is less synchronized, leaving inhibition to be accomplished by strategic control rather than automatically. At the behavioral level, there was no difference between the groups.

\section{Key Words: Autism, factor analysis, fMRI, functional connectivity,} inhibitory control, response inhibition, underconnectivity

$\mathrm{R}$ esponse inhibition is a key executive control process that helps govern complex cognition and in turn complex adaptive behavior. When response inhibition is functioning properly its contributions are not visible, because a successfully inhibited response simply does not emerge, at least not behaviorally. In autism, several types of behavior are commonly observed that are suggestive of malfunctioning of response inhibition processes. The inability to inhibit context-inappropriate behavior is typical of autism and often leads to actions and verbalizations that are inappropriate in timing or to the circumstances. Even when aware of the need to not respond, people with autism may be unable to suppress an inappropriate behavior. This circumstance leads to socially embarrassing incidents for parents and at times to serious legal consequences. Neuropsychologic tests have reported variable results with regard to performance on tests of inhibition in autism.

Some studies of response inhibition have indicated that high-functioning individuals with autism are not impaired at inhibiting simple responses, such as pressing a button for circles but not for squares (Bishop and Norbury 2005; Goldberg et al. 2005; Kleinhans et al. 2005; Ozonoff and Strayer 1997; Ozonoff et al. 1994). Several paradigms have shown no impairments in people with autism in response inhibition, such as Stroop tasks (Eskes et al. 1990; Ozonoff and Jensen 1999; Schmitz et al. 2006), "go-no-go" tasks (Schmitz et al. 2006), simple inhibition in

From the Center for Cognitive Brain Imaging (RKK, TAK, MAJ), Carnegie Mellon University; and Departments of Psychiatry and Neurology (NJM), University of Pittsburgh School of Medicine, Pittsburgh, Pennsylvania.

Address reprint requests to Marcel A. Just, Ph.D., Center for Cognitive Brain Imaging, Department of Psychology, Carnegie Mellon University, Pittsburgh, PA 15213; E-mail: just@cmu.edu.

Received March 3, 2006; revised June 28, 2006; accepted August 4, 2006. "go-no-go" tasks (Ozonoff and Strayer 1997; Ozonoff et al. 1994), stop-signal tasks (Ozonoff and Strayer 1997), negative priming tasks (Brian et al. 2003; Ozonoff and Strayer 1997), and switch tasks (Schmitz et al. 2006). All these paradigms have simple inhibition as the common factor. However, people with autism have difficulty in tasks that impose a working memory load in addition to requiring response inhibition (Hughes 1996; Hughes and Russell 1993; Minshew et al. 1999; Russell 1997) or when they are required to shift from one response set to another (Ozonoff and Strayer 1997; Ozonoff et al. 1994). Paradigms such as memory-based eye movement inhibition tasks (Goldberg et al. 2002, 2005; Luna et al. 2006; Minshew et al. 1999), the set shifting component of "go-no-go" tasks (Ozonoff and Strayer 1997; Ozonoff et al. 1994), and NEPSY Knock-Tap tasks (Korkman et al. 1998) that tap working memory and inhibitory control (Joseph et al. 2005) have shown impairments in performance in autism. Thus, behavioral studies indicate that the inhibition impairments in autism are not ubiquitous but depend on the nature, complexity, and subdomains of the task at hand. The functional magnetic resonance imaging (fMRI) study reported here offers the possibility of determining the neural basis of the inhibition impairment. The study investigated the brain activation and synchronization in autism in a simple response inhibition task as well as in a 1-back inhibition task.

It is important to recognize that response inhibition and working memory are functions that do not occur in isolation but whose expression occurs in the context of some task. For example, in normal participants, the size of a working memory load affects the ability to suppress inappropriate responses (Roberts et al. 1994; Conway et al. 1999). Moreover, response inhibition must be carefully coordinated with other facets of processing, so that just the right response is inhibited at just the right time. A theory of cortical underconnectivity in autism (Just et al. 2004) would suggest that the coordinated nature of response inhibition (requiring communication among the neural centers underpinning a task) might be particularly susceptible to disruption in autism. In particular, intact 
neuronal connectivity is essential for the ability to exert top-down control that allows voluntary response suppression. Investigating response inhibition from an underconnectivity perspective places a focus not only on the regions found to be involved in accomplishing inhibition but also on the coordination among various regions and networks.

The study used a "go-no-go" paradigm, which requires the participants to respond on "go" trials and to inhibit their response on "no-go" trials. Cortical circuits involving diverse areas of frontal cortex and other association cortex sites such as parietal cortex are implicated in the inhibition of responses during "no-go" trials in a "go-no-go" task (Liddle et al. 2001). Brain imaging studies have also found the anterior cingulate cortex to be involved in the detection of conflict between competing responses (Botvinick et al. 2001; Carter et al. 1998) and in monitoring for the occurrence of response conflict in information processing (Barch et al. 2000; Botvinick et al. 1999, 2001; Braver et al. 2001; Carter et al. 1998; Carter et al. 2000). Such findings also suggest that the anterior cingulate would be particularly active during "no-go" events.

Atypical activation in cingulate cortex in autism has been found in several studies. For instance, Gomot et al. (2006) found reduced activation in left anterior cingulate gyrus in autism during auditory detection of acoustic deviance and novelty. Luna et al. (2002) found reduced activation in autism in posterior cingulate cortex in a spatial working memory task. Studies have also suggested that atypical preparation in motor planning tasks in autism is consistent with a disturbance of functions in the supplementary motor cortex and the anterior cingulate (Rinehart et al. 2001). The results from these studies suggest impairments in autism related to cingulate cortex functioning. Some of these functions include monitoring one's performance from time to time and detecting any errors that are made. In addition to considerations of cortical function, differences in brain structure also enter into accounts of autism. Converging evidence from magnetic resonance imaging (MRI)-based morphometry (Abell et al. 1999; Haznedar et al. 2000), diffusion tensor imaging (DTI) (Barnea-Goraly et al. 2004), positron-emission tomography (PET) (Haznedar et al. 1997, 2000), single-photon emission computed tomography (SPECT) (Ohnishi et al. 2000), and postmortem studies (Bauman and Kemper 1994) have indicated abnormalities associated with cingulate cortex in autism.

Response inhibition is a process that needs a good amount of cognitive control and it involves preparing for responding, monitoring performance, and detecting errors. This is accomplished by the coordination of regions like cingulate cortex and other frontal and parietal regions. Based on the previous findings in autism, we hypothesized that participants with autism would show reduced activation in cingulate regions compared with control participants. We also hypothesized that the participants with autism would exhibit lower levels of synchronization of the inhibition network (involving cingulate regions and insula) with frontal and parietal regions. At the behavioral level, we hypothesized that the autism group would show more inhibition-related errors than the control participants in the 1-back inhibition task but not in the simple inhibition task.

\section{Methods and Materials}

\section{Participants}

Twelve high-functioning individuals with autism (mean age 26.8 years) and twelve control participants (mean age 22.5 years) were included in the analyses (Full Scale and Verbal IQ scores of 80 or above based on the Wechsler Abbreviated Scale of Intelligence [WASI]) (Wechsler 1999). Participants were matched
Table 1. Age, IQ, Handedness, and Gender of Participants

\begin{tabular}{|c|c|c|}
\hline Measure & Autism & Control \\
\hline \multicolumn{3}{|l|}{ Age (Years) } \\
\hline Mean \pm SD & $26.8 \pm 7.7$ & $22.5 \pm 3.2$ \\
\hline \multicolumn{3}{|l|}{ VIQ } \\
\hline Mean \pm SD & $110.1 \pm 14.4$ & $114.0 \pm 10.0$ \\
\hline \multicolumn{3}{|l|}{ PIQ } \\
\hline Mean \pm SD & $107.1 \pm 13.8$ & $116.9 \pm 6.4$ \\
\hline \multicolumn{3}{|l|}{ FSIQ } \\
\hline Mean \pm SD & $110.1 \pm 12.6$ & $117.0 \pm 8.7$ \\
\hline \multicolumn{3}{|l|}{ Handedness } \\
\hline Right : left & $11: 1$ & $11: 1$ \\
\hline \multicolumn{3}{|l|}{ Gender } \\
\hline Male : female & $11: 1$ & $11: 1$ \\
\hline
\end{tabular}

$\mathrm{IQ}$, intelligence quotient; VIQ, Verbal Intelligence Quotient; PIQ, Performance Intelligence Quotient; FSIQ, Full-Scale Intelligence Quotient.

on the basis of age and intelligence quotient (IQ) (Table 1). The diagnosis of autism was established using two structured research diagnostic instruments, the Autism Diagnostic InterviewRevised (ADI-R) (Lord et al. 1994) and the Autism Diagnostic Observation Schedule-Generic (ADOS-G) (Lord et al. 2000), supplemented with confirmation by expert clinical opinion. Potential participants with autism were excluded on the basis of an associated disorder, such as fragile-X syndrome or tuberous sclerosis. Potential control participants and participants with autism were also excluded if found to have evidence of birth asphyxia, head injury, or a seizure disorder. Exclusionary criteria were based on history, examination, and chromosomal analysis.

The control participants were medically healthy community volunteers recruited to match the participants with autism on age, full-scale IQ, gender, race, and family of origin socioeconomic status, as measured by the Hollingshead method. Potential control participants were screened by questionnaire, telephone, face-to-face interview, and observation during psychometric testing to determine eligibility. Exclusionary criteria included current or past psychiatric and neurologic disorders, birth injury, developmental delay, school problems, acquired brain injury, learning disabilities, and medical disorders with implications for the central nervous system or those requiring regular medication. Potential control participants were also screened to exclude those with medical illnesses or a family history of autism; developmental cognitive, affective, or anxiety disorders; schizophrenia; obsessive-compulsive disorder; or other neurologic or psychiatric disorder thought to have a genetic component in first-degree relatives.

Each participant signed an informed consent that had been approved by the University of Pittsburgh and Carnegie Mellon University Institutional Review Boards. Prior to testing in the scanner, each participant was familiarized with the task and had as many opportunities to practice in the MRI simulator as needed for comfort and to attain head motion quality standards.

\section{Experimental Paradigm}

This experiment assessed the brain activation and performance in autism and control participants during a response inhibition task. There were three experimental conditions and a fixation baseline condition. In all three experimental conditions, alphabetic characters were displayed one at a time, in the center of the computer screen, at a rate of one every $1000 \mathrm{msec}$. The participants were instructed to press a button with their index 
finger for every letter except for those that met certain criteria. Those criteria for inhibiting the response varied across the three conditions in the experiment.

First, in a baseline condition, participants were instructed to "press for every letter except A"; however, no As were presented here and thus the participant pressed a button for every letter, never requiring any inhibition of a response. The second condition, simple response inhibition, had the same instructions, but in this condition As were presented. Fifteen As were presented per 60 -letter run ( $25 \%$ of the time, i.e., on average, once every $4 \mathrm{sec}$ ). The third condition, 1-back inhibition, displayed only the letters "F" or "G." The participant was instructed to "press for every letter EXCEPT for the second of two consecutive Fs and/or two consecutive Gs" (Figure 1). As in the previous condition, letters requiring inhibition occurred 15 times per 60-letter run (25\% of the time, i.e., on average, once every $4 \mathrm{sec}$ ). The inhibition tasks were developed based on two previous studies (Casey et al. 1997; Garavan et al. 1999).

Each participant practiced the task before going into the scanner. The practice consisted of one 60-letter run of the simple inhibition task and one 60-letter run of the 1-back inhibition task Participants made all responses using a one-button mouse, held in their right hand. The display of each letter lasted $500 \mathrm{msec}$, followed by a 500-msec blank interval. A 6-second delay occurred between 60-letter runs. There were two 60-letter runs of each condition (simple inhibition and 1-back inhibition). In addition, a 24-sec fixation baseline was presented after every two 60 -letter runs to provide a baseline measure of brain activation with which to compare each experimental condition. In this fixation condition, participants fixated on a centered asterisk without performing any task. In addition, one 60-letter run of a no inhibition task (press button for every letter on the screen) was presented at the beginning, which would provide a baseline for contrast with the inhibition conditions. The order of the conditions was counterbalanced.

\section{Imaging Parameters}

The imaging was carried out at the Brain Imaging Research Center (BIRC), University of Pittsburgh and Carnegie Mellon University, on a 3-Tesla Siemens Allegra scanner (Siemens, Erlangen, Germany) using a circularly polarized transmit/receive head coil. The stimuli were rear-projected onto a translucent plastic screen and participants viewed the screen through a mirror attached to the head coil. For the functional imaging, a gradient echo, echo-planar pulse sequence was used with repetition time $(\mathrm{TR})=1000 \mathrm{msec}$, echo time $(\mathrm{TE})=30 \mathrm{msec}$, and a flip angle of $60^{\circ}$. Sixteen adjacent oblique axial slices were

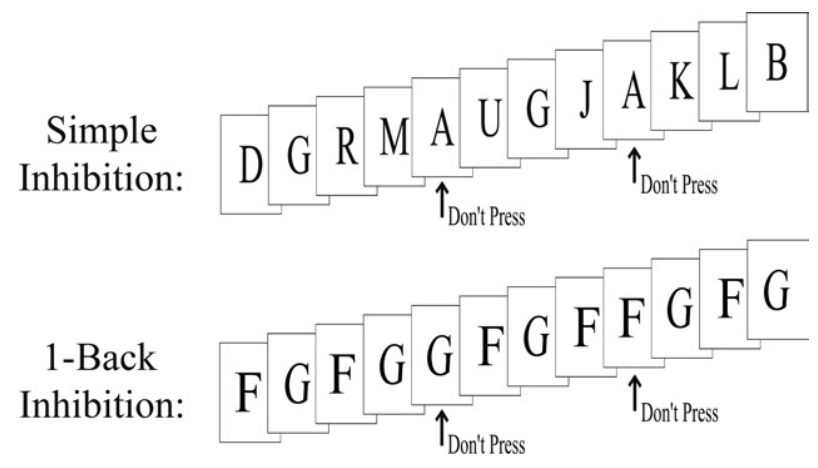

Figure 1. Letter stream stimuli used for the simple response inhibition task and the 1-back response inhibition task. acquired in an interleaved sequence, with 5-mm slice thickness, 1 -mm slice gap, a $20 \times 20 \mathrm{~cm}$ field of view (FOV), and a $64 \times 64$ matrix, resulting in an in-plane resolution of $3.125 \times 3.125 \mathrm{~mm}$. A 160-slice 3D MPRAGE volume scan with $\mathrm{TR}=200 \mathrm{msec}$, TE $=$ $3.34 \mathrm{msec}$, flip angle $=7, \mathrm{FOV}=25.6 \mathrm{~cm}, 256 \times 256$ matrix size, and $1-\mathrm{mm}$ slice thickness was acquired at the same orientation as the oblique axial functional images for each participant.

\section{Distribution of Activation}

To compare the participating groups in terms of the distribution of activation, the data were analyzed using SPM99 software (Wellcome Department of Cognitive Neurology, London, United Kingdom). Images were corrected for slice acquisition timing, motion-corrected, normalized to the Montreal Neurological Institute (MNI) template, resampled to $2 \times 2 \times 2 \mathrm{~mm}$ voxels, and smoothed with an 8-mm Gaussian kernel to decrease spatial noise. Statistical analysis was performed on individual and group data by using the general linear model as implemented in SPM99 (Friston et al. 1995). Group analyses were performed using a random-effects model. Contrasts reflecting the inhibition effects for each group, group by inhibition interactions, and the group differences in the distribution of activation relative to fixation were computed. For the group difference contrasts, possible differences in deactivation (relative to fixation condition) were excluded. An uncorrected height threshold of $p=.005$ and an extent threshold of 10 voxels were used.

\section{Functional Connectivity}

The functional connectivity was computed (separately for each participant) as a correlation between the average time course of signal intensity of all the activated voxels in each member of a pair of regions of interest (ROIs). A total of 21 functional ROIs were defined. Nine ROIs were defined bilaterally, which include inferior frontal gyrus (IFG), middle frontal gyrus (MFG), precentral gyrus (Precen), inferior parietal lobule (IPL), superior parietal lobule (SPL), fusiform gyrus (FG), cingulate gyrus (CINGG), insula, and inferior occipital gyrus (IOG). In addition to these 18 bilateral ROIs, 3 other ROIs were defined: the anterior cingulate gyrus (ACING), the supplementary motor area (SMA), and the right middle temporal gyrus (RMTG). A sphere was defined for each cluster (with a radius from 5 to 10 $\mathrm{mm}$ ) that best captured the cluster of activation in the map for each group. The ROIs used in the analysis were each the union of the six spheres defined for the two groups in each of the three conditions. The activation time course extracted for each participant over the activated voxels within the ROI originated from the normalized and smoothed images, which were low-pass filtered and had the linear trend removed. The time course was extracted at a $t$ threshold of 4.5 , which corresponds to the individual participant's brain activation at a corrected $p$ threshold of .05 , thus making sure that the individual participants showed activation in each defined functional ROI. The defined ROIs had to show at least 12 voxels of activation to be considered for the functional connectivity correlation, i.e., the functional connectivity correlation is between active voxels in two given ROIs. The correlation was computed on the images belonging to all conditions, and it reflects the interaction between the activation in two areas while the participant is performing the task. Fisher's $r$ to $z^{\prime}$ transformation was applied to the correlation coefficients for each participant prior to averaging and statistical comparison of the two groups. Functional connectivity is simply the correlation of activation across regions and does not refer to anatomical connectivity. 


\section{Factor Analysis}

A factor analysis of the functional connectivities was performed to indicate the groupings of the ROIs into networks based on the similarities of their time courses (Koshino et al. 2005). For each ROI pair, mean z'-transformed values of the functional connectivity measures were computed across participants for each group. The mean z'-transformed values were then converted back to correlation coefficients, and a correlation matrix was constructed for each group. The resulting connectivity matrices included 21 functional ROIs. An exploratory factor analysis (e.g., McLaughlin et al. 1992; Peterson et al. 1999) was then performed for each group separately. The logic behind the factor analyses was that each factor would correspond to a large-scale network of brain regions executing some high-level function (Mesulam 1990, 1998). Factor loadings represent the degree to which each of the ROIs correlates with each of the factors, and ROIs that had factor loadings of .5 or greater were taken into consideration in interpreting the results.

\section{Results}

\section{Overview}

The main finding was that the autism group showed a reduced level of brain activation in the simple inhibition condition relative to the control participants primarily in regions associated with inhibition (like middle or anterior cingulate cortex), and in the 1-back inhibition condition, a similar pattern occurred in these areas. In addition, the autism group showed more activation in bilateral premotor regions, areas associated with processing cues in task preparation. The participants with autism also showed reduced functional connectivity relative to the control participants between the anterior cingulate inhibition network and parietal regions during the working memory inhibition condition. At the behavioral level, there was no significant difference between the autism and control participants in performance.

\section{Behavioral Results}

There were no statistically reliable differences between the two groups in behavioral performance. Neither the reaction time (autism mean $=394 \mathrm{msec}$; control mean $=383 \mathrm{msec}$ ) nor the false alarm rate (autism mean $=17.2 \%$; control mean $=19.6 \%$ ) showed any reliable difference between the two groups. The autism group showed a greater effect of working memory load on false alarm rate. The autism group made more false alarm errors in the 1-back inhibition condition (21\%) than in the simple inhibition condition (14\%), whereas the control group showed no reliable difference between the two conditions in false alarm error rates (simple inhibition: 20\%; 1-back inhibition: 19\%), resulting in a reliable interaction between group and condition $[F(1,22)=4.27, p<.05]$. Although the group by task interaction was expected, the pattern of results found here, with the autism group showing equivalent performance in the working memory condition, was not predicted.

\section{Group Differences in Brain Activation}

In the simple inhibition condition, the autism group showed reliably lower brain activation than control participants in several regions previously found to be involved in inhibition. These regions were right insula, right inferior frontal gyrus, right cingulate gyrus, and right premotor cortex (Figure 2 for reduced cingulate activation and Table 2 for the list of all activated regions). There seems to be two main networks involved in accomplishing response inhibition: 1) an inhibition network

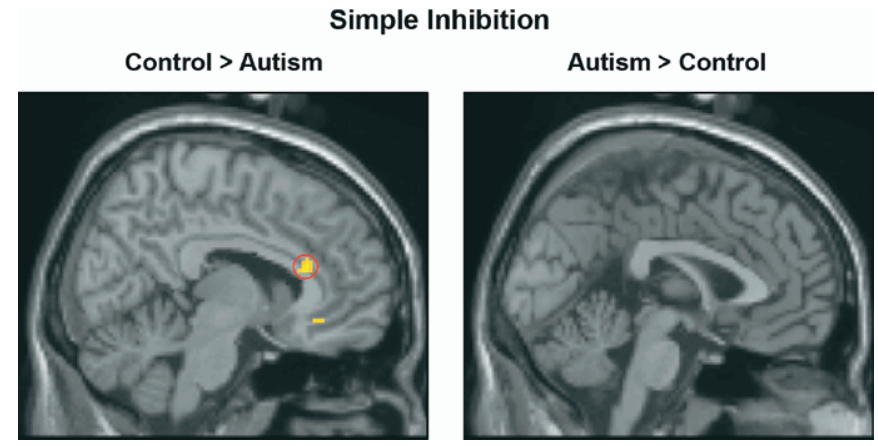

Figure 2. Group subtraction results in the simple inhibition condition. The control group showed greater cingulate activation than the autism group (left panel). The threshold for significant activation was $p<.005$ for a spatial extent of at least 10 voxels, uncorrected for multiple comparisons.

consisting of regions such as the cingulate cortex and insula, and 2) a strategic or executive network involving the prefrontal and parietal regions. The results showed lower activation in autism not only in the inhibition network but also in certain components of the executive network such as the right inferior frontal gyrus.

In the more demanding 1-back inhibition condition, the autism group again showed reliably lower brain activation in several regions, including the left anterior cingulate gyrus, left precuneus, and right angular gyrus (Figure 3 shows reduced cingulate activation and Table 3 has the list of all activated regions). On the other hand, the autism group showed more activation than control participants in left and right premotor regions. This condition is relatively difficult since it has both working memory and inhibition components. Unlike the simple inhibition condition, there was no group difference in right inferior frontal activation in 1-back inhibition. This may be because both groups were activating this region equally to keep up with the demands of working memory in this condition. The behavioral data showed that the autism group made reliably more errors in this condition compared with their performance in simple inhibition.

Unlike the simple inhibition and 1-back inhibition conditions, there was no group difference in activation in the cingulate cortex during the condition where no inhibition was involved. In other words, the cingulate activation difference between the groups emerged only when there was an inhibitory demand, with the autism group showing lower activation than control participants. This group (autism vs. control participants) $\times$ condition (inhibition vs. no inhibition) interaction was significant in the cingulate region $[t(22)=5.43, p<.05]$. Cingulate cortex, especially the anterior cingulate, has been found to be involved in cognitive control processes, including several functions related to response inhibition such as error detection and conflict monitoring.

\section{Factor Analysis}

The main finding in the factor analysis was that in the autism group, unlike the control group, the inhibition network (consisting of anterior cingulate cortex, bilateral cingulate gyri, and bilateral insula) was grouped separately from other frontalparietal areas (the right middle frontal, right inferior frontal, and right inferior parietal) in the 1-back inhibition condition. The inhibition network emerged as an isolated factor. This isolation of the inhibition network in autism is evident from the factor structure table (Figure 4), where the two red boxes indicate how the groups differed in this respect. This inhibition factor is Factor 
Table 2. Group Differences in Activation for Control Participants and Participants with Autism During Simple Response Inhibition

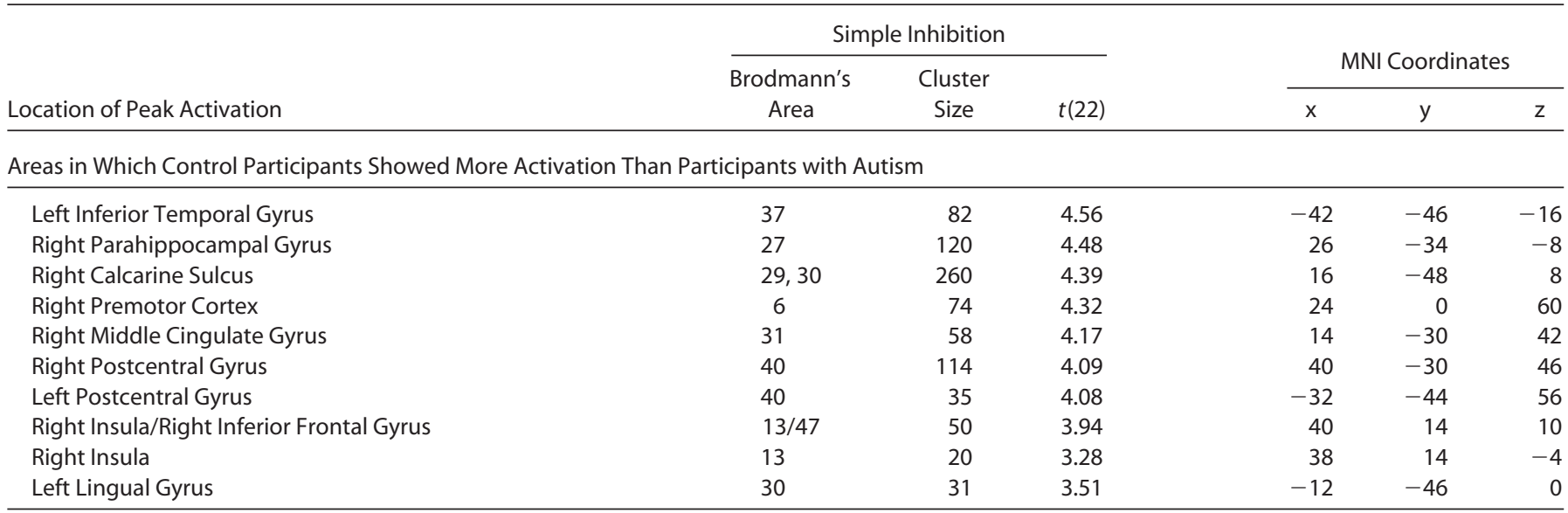

Areas in Which Participants with Autism Showed More Activation Than Control Participants

\section{None}

The threshold for significant activation was $p<.005$ for a spatial extent of at least 10 voxels, uncorrected for multiple comparisons. Region labels apply to the entire extent of the cluster. $t$-values, and MNI coordinates are for the peak activated voxel in each cluster only.

$\mathrm{MNI}$, Montreal Neurological Institute.

2 (F2) for the autism group, which corresponds to Factor 1 (F1) for the control group. The autism group differed from the control participants in that their inhibition network seemed to be working by itself. In control participants, the areas of this network were grouped with right frontal and parietal areas, indicating a greater integration of the inhibition processes with the frontalparietal processes.

Aside from the inhibition factor described above (one of the emerging factors for both groups), both groups had two additional factors that were identical. These factors were 1) the frontal-parietal (green boxes in Figure 4) with bilateral inferior frontal, precentral, inferior parietal, superior parietal, and supplementary motor areas grouped together (F1 for the autism group corresponding to F2 for control participants), and 2) the occipital-temporal (blue boxes in Figure 4) with left and right fusiform and left and right inferior occipital areas grouped together (Factor 3 [F3] for both groups)

\section{Functional Connectivity}

Based on the factor analysis results, a functional connectivity analysis grouped together those regions that belonged to a particular factor, essentially defining a network. This functional

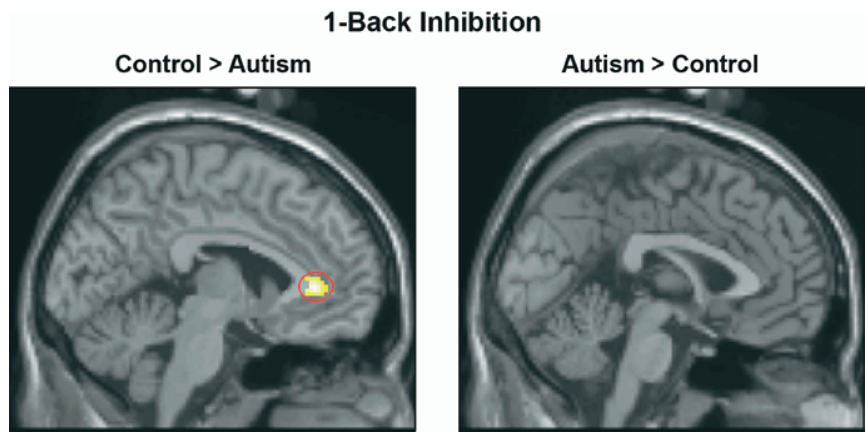

Figure 3. Group subtraction results in 1-back inhibition condition. The control group showed greater cingulate activation than the autism group (left panel). The threshold for significant activation was $p<.005$ for a spatial extent of at least 10 voxels, uncorrected for multiple comparisons. connectivity network analysis revealed that during the 1-back inhibition task, the autism group showed reliably reduced functional connectivity between the inhibition network and right inferior parietal areas, providing converging evidence to the factor analysis results $[t(22)=2.65, p<.05]$. In addition, the inhibition network also showed lower functional connectivity with right inferior frontal gyrus in the autism group compared with control participants $[t(22)=2.03, p<.05]$. In the simple inhibition condition, although the autism group showed the same pattern, the effect was not statistically reliable. In participants with autism, the inhibition network is not as well coordinated with the other networks and regions involved in performing the task.

Based on the prediction that the inhibition network would show underconnectivity with other regions for the autism group relative to the control participants, two planned contrasts comparing the group differences in connectivity between the inhibition network and right inferior frontal gyrus and right inferior parietal lobe were conducted. The results of this analysis of variance (ANOVA) confirmed that the inhibition network was functionally underconnected in the autism group with right inferior parietal lobe $[F(1,22)=7.04, p<.05]$ and with right inferior frontal gyrus $[F(1,22)=4.13, p<.05]$. This functional connectivity difference was reliable only for the 1-back inhibition condition.

\section{Discussion}

This study showed reliable group differences in brain activation in cortical regions involved in inhibitory control. In addition, these regions were found to be functionally underconnected with key frontal and parietal regions in participants with autism. The participants with autism showed reduced activation mainly in one region, the cingulate cortex. This hypoactivation in cingulate regions in autism is interesting considering the role of this region in inhibitory control and the functional and structural abnormalities found by previous studies in the cingulate regions in autism. The cingulate cortex has been found to play a central role in several functions associated with response inhibition, 
Table 3. Group Differences in Activation for Control Participants and Participants with Autism During 1-Back Inhibition Task

\begin{tabular}{|c|c|c|c|c|c|c|}
\hline \multirow[b]{3}{*}{ Location of Peak Activation } & \multicolumn{3}{|c|}{ N-Back Inhibition } & \multirow{2}{*}{\multicolumn{3}{|c|}{ MNI Coordinates }} \\
\hline & \multirow{2}{*}{$\begin{array}{c}\text { Brodmann's } \\
\text { Area }\end{array}$} & \multirow{2}{*}{$\begin{array}{c}\text { Cluster } \\
\text { Size }\end{array}$} & \multirow[b]{2}{*}{$t(22)$} & & & \\
\hline & & & & $\mathrm{x}$ & y & z \\
\hline \multicolumn{7}{|c|}{ Areas in Which Control Participants Showed More Activation Than Participants with Autism } \\
\hline Left Anterior Cingulate Gyrus & 24 & 115 & 4.73 & -4 & 38 & 4 \\
\hline Left Middle Occipital Gyrus & 19,39 & 137 & 4.45 & -40 & -80 & 34 \\
\hline Left Calcarine & 30 & 257 & 4.43 & -16 & -54 & 12 \\
\hline Right Calcarine & 29 & 100 & 3.48 & 16 & -50 & 10 \\
\hline Right Angular Gyrus & 39 & 31 & 3.99 & 48 & -72 & 32 \\
\hline Left Precuneus & 7 & 41 & 3.46 & -8 & -64 & 32 \\
\hline
\end{tabular}

Areas in Which Participants with Autism Showed More Activation Than Control Participants

\begin{tabular}{llrrrr}
\hline Left Premotor & 6 & 26 & 4.29 & -28 & -4 \\
Right Premotor & 9 & 50 & 3.44 & 56 & 38 \\
\hline
\end{tabular}

The threshold for significant activation was $p<.005$ for a spatial extent of at least 10 voxels, uncorrected for multiple comparisons. Region labels apply to the entire extent of the cluster. $t$-values and MNI coordinates are for the peak activated voxel in each cluster only.

MNI, Montreal Neurological Institute.

such as monitoring task performance, conflict monitoring, and error detection (Botvinick et al. 2004; Bush et al. 2000). All these functions are part of the type of complex information processing in which people with autism have difficulty (Minshew et al. 1997). According to Minshew et al. (2002), autism may reflect a disturbance in resolving conflict between different strategies and monitoring and switching strategies to achieve goals. Lower levels of cingulate activation in participants with autism might be the source of difficulty in these functions. Morphometric studies have found anterior cingulate in autism to be reliably smaller than the control participants (Haznedar et al. 1997, 2000). A diffusion tensor imaging study found reduced fractional anisotropy in anterior cingulate cortex in autism (Barnea-Goraly et al. 2004). The finding of hypoactivation in the cingulate region in the present study adds further evidence to the atypical functioning associated with this region in autism. But as we note below, the breadth of the activation abnormalities in autism makes it unlikely that any one region is the single source of the problem. We ultimately attribute the abnormal activation to interregional underconnectivity, particularly between frontal and other regions.

Another source of evidence of lower inhibitory control in autism is related to the reduced activation in the anterior insula. This may indicate not only the difficulties people with autism have in controlling attention but also in executive planning to accomplish the task. Insula is commonly activated in tasks that require executive control of attention, including those that require manipulation of information in working memory (Wager and Smith 2003), response inhibition, shifting attention (Wager et al. 2004), and suppression of conscious thoughts (Wyland et al. 2003). The insula is closely connected to the prefrontal cortex and anterior cingulate gyrus and forms part of a frontal-striatal attentional network (Schmitz et al. 2006). The hypoactivation in insular cortex along with cingulate cortex in autism suggests a disordering of inhibitory control in the task. In the only published neuroimaging study of response inhibition in autism, Schmitz et al. (2006) found increased activation in people with autism spectrum disorders in left inferior frontal areas during motor inhibition and in left insula during the Stroop task. The participants in that study were diagnosed with Asperger Syndrome (AS), unlike the high-functioning individuals with autism in our study. The increased activation in AS in their study may not be related to inhibition per se since the activation was in the left hemisphere. It may be possible that the AS participants (who have no language delay and are generally verbal) in that study tended to use internal verbalization during the inhibition task, hence the increased left inferior frontal gyrus activation. The increased insula activation in their study was again in the left hemisphere (for the Stroop task). Left insula also has been found to be active when participants do subvocal rehearsal (Paulesu et al. 1996).

Behaviorally, the participants with autism performed as well as the control participants in the simple inhibition condition; however, they made more errors in 1-back inhibition. In other words, when response inhibition had an additional working memory component, there was a decline in the performance of participants with autism. The addition of a working memory component may have undermined the effectiveness of any

\begin{tabular}{|c|c|c|c|c|c|c|c|c|}
\hline \multirow[b]{2}{*}{ Factor } & \multicolumn{4}{|l|}{ Autism } & \multicolumn{4}{|l|}{ Control } \\
\hline & F1 & $\mathrm{F} 2$ & $\mathrm{~F} 3$ & & $\mathrm{~F} 1$ & $\mathrm{~F} 2$ & F3 & \\
\hline Anterior cingulate gyrus & . & 0.80 & . & & 0.84 & . & . & \\
\hline $\mathrm{L}$ cingulate gyrus & . & 0.73 & . & & 0.68 & . & . & \\
\hline $\mathrm{R}$ cingulate gyrus & . & 0.80 & . & & 0.85 & . & . & \\
\hline L insula & . & 0.54 & . & & 0.56 & . & . & \\
\hline $\mathrm{R}$ insula & . & 0.50 & . & & 0.60 & . & . & \\
\hline $\begin{array}{l}\text { Supplementary motor } \\
\text { area }\end{array}$ & 0.56 & 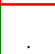 & . & & & 0.51 & . & \\
\hline $\mathrm{L}$ inferior frontal gyrus & 0.65 & . & . & & . & 0.60 & . & \\
\hline$R$ inferior frontal gyrus & 0.71 & . & . & & . & 0.55 & . & \\
\hline $\mathrm{L}$ middle frontal gyrus & . & . & . & & . & & . & \\
\hline $\mathrm{R}$ middle frontal gyrus & . & . & . & & 0.50 & & . & \\
\hline L precentral gyrus & 0.56 & . & . & & . & 0.50 & . & \\
\hline $\mathrm{R}$ precentral gyrus & 0.68 & . & . & & . & 0.61 & . & \\
\hline $\mathrm{L}$ intraparietal sulcus & 0.72 & . & . & & 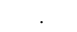 & 0.73 & . & \\
\hline $\mathrm{R}$ intlraparietal sulcus & 0.73 & . & . & & 0.52 & 0.58 & . & \\
\hline L superior parietal lobe & 0.71 & . & . & & . & 0.75 & . & \\
\hline $\mathrm{R}$ superior parietal lobe & 0.68 & & - & & . & 0.70 & & \\
\hline $\mathrm{L}$ fusiform gyrus & . & . & 0.75 & & . & . & 0.70 & \\
\hline $\mathrm{R}$ fusiform gyrus & . & . & 0.65 & & . & . & 0.70 & \\
\hline $\mathrm{R}$ middle temporal gyrus & . & . & . & & . & . & . & \\
\hline $\mathrm{L}$ inferior occipital gyrus & . & 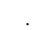 & 0.80 & & . & & 0.70 & \\
\hline $\mathrm{R}$ inferior occipital gyrus & . & . & 0.68 & Total & & . & 0.74 & Total \\
\hline Eigen value & 4.82 & 3.58 & 2.95 & 11.35 & 4.61 & 4.45 & 2.94 & 12.00 \\
\hline$\%$ variance explained & & & & 54.03 & & & & 57.16 \\
\hline F1: Frontal and parietal & & & & & $\begin{array}{l}\text { F1: Inhil } \\
\text { parietal }\end{array}$ & Rigl & frontal & \\
\hline F2: Inhibition & & & & & F2: Fron & ad parie & & \\
\hline F3: Occipital and inferior & aporal & & & & F3: Occi & and inf & rior te & aporal \\
\hline
\end{tabular}

Figure 4. Factor analysis. 
coping strategy that people with autism may have used in the simple inhibition condition.

\section{Functional Connectivity}

Although the anterior cingulate cortex (ACC) plays an important role in inhibitory control, there may be other cortical regions working in synchrony with ACC in accomplishing inhibitory control. The results from factor analysis and functional connectivity analysis in the present study showed a reliably lower degree of synchronization and coordination between key cortical networks in autism compared with the control group. Weaker integration was found in the connections between the ACC inhibition system (consisting of regions such as anterior cingulate gyrus, middle cingulate gyrus, and insula) and the right frontalparietal inhibition system (right inferior frontal, middle frontal, and right inferior parietal regions). While the ACC system was functioning out of synchrony with other networks in the autism group, this system was synchronized with right frontal and parietal regions in the control participants. According to Garavan et al. (2002), the ACC system is involved in relatively faster and urgent inhibition, whereas the frontal-parietal system is involved in more deliberate and controlled inhibition. In control participants, these two inhibition systems seem to work together as an integrated system recruiting more attentional resources. The inferior parietal regions, in conjunction with activation in ACC, have been related to error detection (Carter et al. 1998), response conflict (Braver et al. 2001; van Veen et al. 2001), and to visual-spatial alerting and orienting (Corbetta et al. 2000; Coull et al. 1996). All these functions are part of constructing and executing metacognitive strategies and having inhibitory control.

While several studies have explored the role of prefrontal cortex in cognitive control (Miller and Cohen 2001 has a review), the functional connectivity between cognitive control in prefrontal cortex and other brain regions has not been addressed. Orbitofrontal and anterior cingulate cortex have bilateral cortical connections within ventral and dorsal prefrontal cortex, insula, and parietal cortex, as well as with subcortical connections with amygdala, striatum, and thalamus (Cavada et al. 2000; Musil and Olson 1988; Vogt and Pandya 1987). Egner and Hirsch (2005) recently explored the functional integration in cognitive control and found that the activation in prefrontal regions was accompanied by increased functional integration with right temporal and parietal areas. The lower functional connectivity between the ACC system and right hemisphere frontal and parietal regions in autism in our study provides an important insight into the difference in inhibitory control between the two groups in this task. It should be noted here that the activation and connectivity differences are occurring despite no behavioral difference between the groups. That gives further evidence to the fact that the differences between the two groups are at a finer level, with the autism group accomplishing the task always through a different route. Some studies have reported that people with autism have abnormalities in prefrontal and anterior cingulate cortex metabolism (Carper and Courchesne 2005; Horwitz et al. 1988; Siegel et al. 1995) and anatomical abnormalities in regions involved in inhibition (Schmitz et al. 2006). Allman et al. (2001) suggested that the spindle cell structures of the anterior cingulate may serve to connect widespread areas of the brain to achieve the synchronization of information in difficult problem-solving situations. Structural and functional abnormalities in the cingulate cortex in autism could be symptomatic of a lower level of connectivity in autism.
It should be noted here that the reduced functional connectivity in autism does not result from reduced activation. First and most importantly, in the vast majority of the ROI pairs in which underconnectivity was observed in autism, there was no reduction of activation in autism. Second, in the case where there was lower activation in the autism group (involving anterior cingulate cortex), the underconnectivity was found between a network (set of ROI pairs), which included several other areas such as middle cingulate, insula, and right parietal region. In these other areas, there was no activation difference between the groups, and still these ROIs showed lower functional connectivity with the right parietal region in the autism group. Third, other studies have found underconnectivity in several individual ROI pairs where there was no reduction in activation, such as a study on executive functioning which found, despite having an equal amount of activation in autism and control groups, that there was widespread functional underconnectivity in the autism group (Just et al, in press). The findings strongly indicate that underconnectivity does not stem from a reduction in activation.

Successfully withholding a response to the "no-go" trials is argued to represent inhibitory control over a prepotent response, typically resulting in activation of prefrontal, parietal (predominantly right hemisphere), and midline (ACC and pre-SMA) regions (Garavan et al. 2002; Liddle et al. 2001; Rubia et al. 2003; Watanabe et al. 2002). These regions interact, coordinate, and function as a unit to accomplish inhibition. Because of the cortical underconnectivity in autism, the interregional coordination may be particularly stressed in complex tasks, such as 1-back inhibition in the present study, that involve creating a novel strategy, flexibility, and monitoring performance. A novel task requires the underpinning brain regions to dynamically configure themselves into an appropriate network, and the poorer connectivity in autism impairs this dynamic ability. The compensatory strategy that often arises in autism under such circumstances is a reversion to relying on lower level perceptually based strategies that require less connectivity to frontal areas.

Reduced functional connectivity in people with autism has been found in diverse tasks, such as sentence comprehension (Just et al. 2004) and verbal working memory (Koshino et al. 2005). Findings from these studies provide evidence for the generality of the underconnectivity across tasks, as well as the specificity of the underconnectivity to frontal cortical regions. The larger connectivity differences were found mainly in the long-distance connections between frontal and other regions. Recently, we found frontal-parietal underconnectivity in an executive function task (Just et al, in press) and in a language comprehension task involving visual-spatial imagery (Kana et al. 2006). This is consistent with other studies that found reduced long-distance structural (Courchesne and Pierce 2005) and functional (Castelli et al. 2002) connectivity.

In conclusion, the findings of this study suggest that in individuals with autism, inhibitory processes do not function as part of a coordinated and synchronized cortical network. Future neuroimaging studies of autism may further modify this account, explaining the breadth and the specificity of the atypical inhibitory function in autism.

This research was supported by the Collaborative Program of Excellence in Autism (CPEA) Grant HD35469 from the National Institute of Child Health and Human Development and the Cure Autism Now Young Investigator Award. 
We thank Sarah Schipul and Stacey Becker for assistance with the data collection and data analysis and Beatriz Luna and Diane Williams for providing useful comments on an earlier version of the manuscript. We also thank the research participants for their participation and commitment.

Abell F, Krams M, Ashburner J, Passingham R, Friston K, Frackowiak R, et al. (1999): The neuroanatomy of autism: A voxel-based whole brain analysis of structural scans. Neuroreport 10:1647-1651.

Allman JM, Hakeem A, Erwin JM, Nimchinsky E, Hof P (2001): The anterior cingulate cortex: The evolution of an interface between emotion and cognition. Ann N Y Acad Sci 935:107-117.

Barch DM, Braver TS, Sabb FW, Noll DC (2000): Anterior cingulate and the monitoring of response conflict: Evidence from an fMRI study of overt verb generation. J Cogn Neurosci 12:298-309.

Barnea-Goraly N, Kwon H, Menon V, Eliez S, Lotspeich L, Reiss AL (2004): White matter structure in autism: Preliminary evidence from diffusion tensor imaging. Biol Psychiatry 55:323-326.

Bauman ML, Kemper TL (1994): Neuroanatomic observations of the brain in autism. In: Bauman ML, Kemper TL, editors. The Neurobiology of Autism. Baltimore: Johns Hopkins Press, 119-145.

Bishop DVM, Norbury CF (2005): Executive functions in children with communication impairments, in relation to autistic symptomatology 2: Response inhibition. Autism 9:29-43.

Botvinick MM, Braver TS, Barch DM, Carter CS, Cohen JD (2001): Conflict monitoring and cognitive control. Psychol Rev 108:624-652.

Botvinick MM, Cohen JD, Carter CS (2004): Conflict monitoring and anterior cingulate cortex: An update. Trends Cogn Sci 8:539-546.

Botvinick MM, Nystrom LE, Fissell K, Carter CS, Cohen JD (1999): Conflict monitoring versus selection-for-action in anterior cingulate cortex. $\mathrm{Na}$ ture 402:179-181.

Braver S, Barch D, Kelley W, Buckner R, Cohen N, Miezin F, et al. (2001): Direct comparison of prefrontal cortex regions engaged by working and longterm memory tasks. Neuroimage 14:48-59.

Brian JA, Tipper SP, Weaver B, Bryson SE (2003): Inhibitory mechanisms in autism spectrum disorders: Typical selective inhibition of location versus facilitated perceptual processing. J Child Psychol Psychiatry 44:552-560.

Bush G, Luu P, Posner MI (2000): Cognitive and emotional influences in anterior cingulate cortex. Trends Cogn Sci 4:215-222.

Carper RA, Courchesne E (2005): Localized enlargement of the frontal cortex in early autism. Biol Psychiatry 57:126-133.

Carter CS, Braver TS, Barch DM, Botvinick MM, Noll D, Cohen JD (1998): Anterior cingulate cortex, error detection and the online monitoring of performance. Science 280:747-749.

Carter CS, MacDonald AM, Botvinick MM, Ross LL, Stenger VA, Noll D, et al. (2000): Parsing executive processes: Strategic versus evaluative functions of the anterior cingulate cortex. Proc Natl Acad Sci U S A 97:19441948.

Casey BJ, Trainor RJ, Orendi JL, Schubert AB, Nystrom LE, Giedd JN, et al. (1997): A developmental functional MRI study of prefrontal activation during performance of a go-no-go task. J Cogn Neurosci 9:835-847.

Castelli F, Frith C, Happé F, Frith U (2002): Autism, Asperger syndrome and brain mechanisms for the attribution of mental states to animated shapes. Brain 125:1839-1849.

Cavada C, Company T, Tejedor J, Cruz-Rizzolo RJ, Reinoso-Suarez F (2000): The anatomical connections of the macaque monkey orbitofrontal cortex. A review. Cereb Cortex 10:220-242.

Conway AR, Tuholski SW, Shisler RJ, Engle RW (1999): The effect of memory load on negative priming: An individual differences investigation. Mem Cogn 27:1042-1050.

Corbetta M, Kincade JM, Ollinger JM, McAvoy MP, Shulman GL (2000): Voluntary orienting is dissociated from target detection in human posterior parietal cortex. Nat Neurosci 3:292-297.

Coull JT, Frith CD, Frackowiak RS, Grasby PM (1996): A fronto-parietal network for rapid visual information processing: A PET study of sustained attention and working memory. Neuropsychologia 34:1085-1095.

Courchesne E, Pierce K (2005): Why the frontal cortex in autism might be talking only to itself: Local over-connectivity but long-distance disconnections. Curr Opin Neurobiol 15:225-230.

Egner T, Hirsch J (2005): The neural correlates and functional integration of cognitive control in a Stroop task. Neuroimage 24:539-547.
Eskes GA, Bryson SE, McCormick TA (1990): Comprehension of concrete and abstract words in autistic children. J Autism Dev Disord 20:61-73.

Friston K, Ashburner J, Frith C, Poline J-B, Heather J, Frackowiak R (1995): Spatial registration and normalization of images. Hum Brain Mapp 2:165-189.

Garavan H, Ross TJ, Murphy K, Roche RAP, Stein EA (2002): Dissociable executive functions in the dynamic control of behavior: Inhibition, error detection, and correction. Neuromage 17:1820-1829.

Garavan H, Ross TJ, Stein EA (1999): Right hemispheric dominance of inhibitory control: An event-related functional MRI study. Proc Natl Acad Sci USA 96:8301-8306.

Goldberg MC, Lasker AG, Zee DS, Garth E, Landa RJ, Tien A, et al. (2002): Deficits in the initiation of eye movements in the absence of a visual target in adolescents with high-functioning autism. Neuropsychologia 40:2039-2049.

Goldberg MC, Mostofsky SH, Cutting LE, Mahone EM, Astor BC, Denckla MB, et al. (2005): Subtle executive impairment in children with autism and children with ADHD. J Autism Dev Disord 35:279-293.

Gomot M, Bernard FA, Davis MH, Belmonte MK, Ashwin C, Bullmore ET, et al. (2006): Change detection in children with autism: An auditory eventrelated fMRI study. Neuroimage 29:475-484.

Haznedar MM, Buchsbaum MS, Metzger M, Solimando A, Spiegel-Cohen J, Hollander E (1997): Anterior cingulated gyrus volume and glucose metabolism in autistic disorder. Am J Psychiatry 154:1047-1050.

Haznedar MM, Buchsbaum MS, Wei TC, Hof PR, Cartwright C, Bienstock CA, et al. (2000): Limbic circuitry in patients with autism spectrum disorders studied with positron emission tomography and magnetic resonance imaging. Am J Psychiatry 157:1994-2001.

Horwitz B, Rumsey JM, Grady CL, Rapoport SI (1988): The cerebral metabolic landscape in autism: Intercorrelations of regional glucose utilization. Arch Neurol 45:749-755.

Hughes C (1996): Brief report: Planning problems in autism at the level of motor control. J Autism Dev Disord 26:99-107.

Hughes C, Russell J (1993): Autistic children's difficulty with mental disengagement from an object: Its implications for theories of autism. Dev Psychol 29:498-510.

Joseph RM, McGrath LM, Tager-Flusberg H (2005): Executive dysfunction and its relation to language ability in verbal school-age children with autism. Dev Neuropsychol 27:361-378.

Just MA, Cherkassky VL, Keller TA, Minshew NJ (2004): Cortical activation and synchronization during sentence comprehension in high-functioning autism: Evidence of underconnectivity. Brain 127:1811-1821.

Just MA, Keller TA, Kana RK, Cherkassky V, Carpenter PA, Minshew NJ (in press): Executive processing in the Tower of London puzzle in highfunctioning autism.

Kana RK, Keller TA, Cherkassky VL, Minshew NJ, Just MA (2006): Sentence comprehension in autism: Thinking in pictures with decreased functional connectivity. Brain 129(Pt 9):2484-2493.

Kleinhans N, Akshoomoff N, Delis DC (2005): Executive functions in autism and Asperger's disorder: Flexibility, fluency, and inhibition. Dev Neuropsychol 27(3):379-401.

Korkman M, Kirk U, Kemp S (1998): NEPSY: A Developmental Neuropsychological Assessment. San Antonio, TX: Psychological Corporation.

Koshino H, Carpenter PA, Minshew NJ, Cherkassky VL, Keller TA, Just MA (2005): Functional connectivity in an fMRI working memory task in highfunctioning autism. Neuroimage 24:810-821.

Liddle PF, Kiehl KA, Smith AM (2001): Event-related fMRI study of response inhibition. Hum Brain Mapp 12:100-109.

Lord C, Risi S, Lambrecht L, Cook EH Jr, Leventhal BL, DiLavore PC, et al. (2000): The Autism Diagnostic Observation Schedule-Generic: A standard measure of social and communication deficits associated with the spectrum of autism. J Autism Dev Disord 30:205-223.

Lord C, Rutter M, LeCouteur A (1994): Autism Diagnostic Interview-Revised: A revised version of a diagnostic interview for caregivers of individuals with possible pervasive developmental disorders. J Autism Dev Disord 24:659-685

Luna B, Doll BS, Hegedus SJ, Minshew NJ, Sweeney JA (2006): Maturation of executive function in autism. Biol Psychiatry [Epub ahead of print].

Luna B, Minshew NJ, Garver KE, Lazar NA, Thulborn KR, Eddy WF, et al. (2002): Neocortical system abnormalities in autism. Neurology 59:834-840.

McLaughlin T, Steinberg B, Christensen B, Law I, Parving A, Friberg L (1992): Potential language and attentional networks revealed through factor 
analysis of rCBF data measured with SPECT. J Cereb Blood Flow Metab 12:535-545.

Mesulam MM (1990): Large-scale neurocognitive networks and distributed processing for attention, language and memory. Ann Neurol 28:597613.

Mesulam MM (1998): From sensation to cognition. Brain 121:1013-1052.

Miller EK, Cohen JD (2001): An integrative theory of prefrontal cortex function. Annu Rev Neurosci 24:67-202.

Minshew NJ, Goldstein G, Siegel D (1997): Neuropsychologic functioning in autism: Profile of a complex information processing disorder. J Int Neuropsychol Soc 3:303-316.

Minshew NJ, Luna B, Sweeney JA (1999): Oculomotor evidence for neocortical systems but not cerebellar dysfunction in autism. Neurology 52: 917-922.

Minshew NJ, Meyer J, Goldstein G (2002): Abstract reasoning in autism: A dissociation between concept formation and concept identification. Neuropsychology 16:327-334.

Musil SY, Olson CR (1988): Organization of cortical and subcortical projections to medial prefrontal cortex in cat. J Comp Neurol 272:219-241.

Ohnishi T, Matsuda H, Hashimoto T, Kunihiro T, Nishikawa M, Uema T, et al. (2000): Abnormal regional cerebral blood flow in childhood autism. Brain 123:1838-1844.

Ozonoff S, Jensen J (1999): Brief report: Specific executive function profiles in three neurodevelopmental disorders. J Autism Dev Disord 29:171-177.

Ozonoff S, Strayer DL (1997): Inhibitory function in nonretarded children with autism. J Autism Dev Disord 27:59-77.

Ozonoff S, Strayer DL, McMahon WM, Filloux F (1994): Executive function abilities in autism and Tourette syndrome: An information processing approach. J Child Psychol Psychiatry 35:1015-1032.

Paulesu E, Frith U, Snowling M, Gallagher A, Morton J, Frackowiak R, et al. (1996): Is developmental dyslexia a disconnection syndrome? Brain 119: 143-157.

Peterson BS, Skudlarski P, Gatenby JC, Zhang H, Anderson AW, Gore JC (1999): An fMRI study of Stroop word-color interference: Evidence for cingulate subregions subserving multiple distributed attentional systems. Biol Psychiatry 45:1237-1258.
Rinehart NJ, Bradshaw JL, Moss SA, Brereton AV, Tonge BJ (2001): A deficit in shifting attention present in high-functioning autism but not Asperger's disorder. Autism 5:67-80.

Roberts RJ, Hager LD, Heron C (1994): Prefrontal cognitive processes: Working memory and inhibition in the antisaccade task. J Exp Psychol 123: 374-393.

Rubia K, Smith AB, Brammer MJ, Taylor E (2003): Right inferior prefrontal cortex mediates response inhibition while mesial prefrontal cortex is responsible for error detection. Neuroimage 20:351-358.

Russell J (1997): How executive disorders can bring about an inadequate 'theory of mind.' In: Russell J, editor. Autism as an Executive Disorder. Oxford, England: Oxford University Press, 256-304.

Schmitz N, Rubia K, Daly E, Smith A, Williams S, Murphy DGM (2006): Neural correlates of executive function in autistic spectrum disorders. Biol Psychiatry 59:7-16.

Siegel BJ Jr, Nuechterlein KH, Abel L, Wu JC, Buchsbaum MS (1995): Glucose metabolic correlates of continuous performance test performance in adults with a history of infantile autism, schizophrenics, and controls. Schizophr Res 17:85-94.

van Veen V, Cohen JD, Botvinick MM, Stenger VA, Carter CS (2001): Anterior cingulate cortex, conflict monitoring, and levels of processing. Neuroimage 14:1302-1308.

Vogt BA, Pandya DN (1987): Cingulate cortex of the rhesus monkey. II. Cortical afferents. J Comp Neurol 262:271-289.

Wager TD, Reading S, Jonides J (2004): Neuroimaging studies of shifting attention: A meta-analysis. Neuroimage 22:1679-1693.

Wager TD, Smith EE (2003): Neuroimaging studies of working memory: A meta-analysis. Cogn Affect Behav Neurosci 3:255-274.

Watanabe J, Sugiura M, Sato K, Sato Y, Maeda Y, Matsue Y, et al. (2002): The human prefrontal and parietal association cortices are involved in no-go performances: An event-related fMRI study. Neuroimage 17:1207-1216.

Wechsler D (1999): Wechsler Abbreviated Scale of Intelligence (WASI). San Antonio, TX: Psychological Corporation.

Wyland C, Kelley WM, Macrae NC, Gordon H, Heatherton TF (2003): Neural correlates of thought suppression. Neuropsychologia 41:18631867. 